\title{
IMPORTANCIA DE LA TEORÍA DE LA TRANSICIÓN DEMOGRÁFICA PARA PAÍSES EN DESARROLLO
}

\author{
Michael S. Teitelbaum* \\ Nuffild College, Oxford \\ $y$ \\ Oxford University
}

\begin{abstract}
La teorí. ofrece sólo una explicación parcial de las tendencias europeas y orientación ambigüa para países en desarrollo.
\end{abstract}

EN LA ACTUALIDAD, la teoría de la transición demográfica constituye un conocido rasgo distintivo en las discusiones sobre el fenómeno de la población humana, y un elemento reciente de las políticas internacionales. En los debates de la Conferencia Mundial de Población, celebrada en Bucarest en 1974, la teoría de la transición demográfica fue un participante activo, aun cuando de manera implícita. Se dejaron atrás algunos de los sentimientos más atractivos y confiables expresados ("Cuidar de la gente y la población será cuidar de sí mismo", "el desarrollo es el mejor - contraceptivo"), y no hay razón para pensar que los postulantes de estos puntos de vista consideraran que defendían algo que no fuera el conocimiento manifiesto de la ciencia demográfica. ${ }^{1}$

No obstante, la adopción popular de una teoría científica deja muy atrás la elaboración de la teoría misma. La teoría de la transición demográfica fue originalmente desarrollada hace aproximadamente medio siglo y, de manera irónica, su capacidad de explicación y de predicción apareció con la creciente duda científica en los momentos en que estaba logrando la mayor aceptación entre los no científicos. En los círculos científicos esta teoría sólo ha logrado un modesto apoyo como explicación de las experiencias demográficas reales, de las cuales se derivó originalmente la

* Se agradecen los valiosos comentarios de muchos colegas, que por desgracia son demasiado numerosos para mencionarlos aquí a todos. El Estudio sobre Fecundidad Europea de la Oficina de Investigación de la Población y de la Universidad de Princeton, es apoyado por el Instituto Nacional de Salud Infantil y Desarrollo Humano.

${ }^{1}$ Un informe completo sobre los debates se presenta en W. P. Mauldin, N. Choucri, F. W. Notestein, M. S. Teitelbaum, "A Report on Bucharest", Study Family Planning, Nueva York, Population Council, 1974, Núm. 5, p. 357. Para un examen de la asombrosa variedad existente de puntos de vista sobre población y desarrollo, véase M. S. Teitelbaum, Foreign Affairs, 1974, Núm. 52, p. 742 . La importancia de las teorías sobre la relación entre fecundidad y desarrollo para propósitos de política se discute en H. Leibestein, International Labour Review, 1974, 109, p. 443. 
teoría -las de Europa del siglo XIX. La capacidad de explicación y de predicción de esta teoría es susceptible de cuestionamientos científicos adicionales cuando se aplica a las circunstancias sociales y económicas actuales, en extremo diferentes, de Asia, Africa y América Latina.

Por lo tanto, el crédito dado a las afirmaciones basadas en la teoría de la transición, en el sentido de que el desarrollo "se hará cargo de" los . problemas poblacionales, como sucedió en Europa, justifican la afirmación de que se tiene conocimiento: i) acerca de lo que sucedió en Europa; y ii) del grado en el que el mismo proceso es factible en los países en desarrollo, dadas las semejanzas y diferencias entre países de Europa del siglo XIX.

\section{LA TEORÍA DE LA TRANSICIÓN DEMOGRÁFICA}

La teoría $a^{2}$ de la transición demográfic $a^{3}$ constituye una interpretación descriptiva de las transformaciones que tuvieron lugar en las pautas demográficas europeas durante el siglo xIX. Caracteriza tres "etapas" de niveles de fecundidad y mortalidad a las que considera derivadas de los cambios sociales y económicos fundamentales del "desarrollo" o "modernización".

De acuerdo con esta teoría, la etapa I de la transición es aquella que ha caracterizado a las especies humanas durante la mayor parte de su historia - un equilibrio del tamaño de la población en el largo plazo logrado por altas tasas de natalidad y altas tasas de mortalidad. La mortalidad infantil es elevada y de manera similar la fecundidad, aunque quizás nunca al nivel biológico máximo de las especies. La elevada tasa de mortalidad se considera inevitable en ausencia de métodos sanitarios, agricultura, transportes y medicina modernos. Dada esta elevada tasa de mortalidad, por definición se requiere una tasa similar de nacimientos para cualquier población que ha persistido. Para mantener una fecundidad elevada, las sociedades que se encuentran en la etapa I de la transición demográfica, se caracterizan por normas pronatalistas muy acentuadas, apoyadas por valores populares tan sagrados como seculares y reforzadas de manera efectiva por diversas sanciones sociales, es decir por un pronatalismo institucionalizado en alto grado y de cambio lento.

\footnotetext{
"En este artículo el término "teoría" se utiliza en forma restringida dado que puede argumentarse que la teoría de la transación demográfica, en estricto sentido científico, no constituye una teoría.

${ }^{3}$ Las generalizaciones de la teoría de la transición demográfica; desarrolladas en forma gradual a partir del trabajo de diversos expertos, incluído $W$. S. Thompson (véase American Journal of Sociology, 1929, Núm. 34, p. 959) y A. Landry, A. M. Carr-Saunders, F. W. Notestein y C. P. Blacker, citados por D. V. Glass, en Public Healt and Population Change, M. C. Sheps y J. C. Ridley (Comps.), Pittsburg, University of Pittsburg Press, 1965, pp. 13-14. F. W. Notestein presenta resúmenes más extensos en Proceedings of the Eighth International Conference on Agricultural Economics, Londres, Oxford University Press, 1953, pp. 15-31; y G. J. Stolnitz en Population: The Vital Revolution, R. Freedman (Comp.), Nueva York, DoubledayAnchor, 1964, pp. 30-46.
} 
En contraste con el mantenimiento social estable de una elevada tasa de nacimientos, la teoría de la transición contempla el control de la tasa de mortalidad fuera del alcance de las sociedades preindustriales. De cualquier modo, dado que la mayoría de la gente desea salud y una vida prolongada, los nuevos métodos para reducir la mortalidad se adoptan con facilidad a medida que se conocen, lo que da como resultado una disminución gradual de la mortalidad. Tanto la elevada fecundidad como la reducida mortalidad son consideradas benéficas. En este sentido, los factores que apoyan las tasas de nacimiento y de muerte elevadas, son asimétricos y el equilibrio de la etapa I es potencialmente inestable.

Esta inestabilidad conduce a la etapa II de la transación demográfica, la cual se caracteriza por una mortalidad que declina y una fecundidad que permanece a los elevados niveles previos sujetos al control de las instituciones sociales tradicionales. Esta es la etapa de la "explosión demográfica", o sea el rápido crecimiento te la población que resulta de un desbalanceo entre tasas de nacimiento y de muerte.

Por último, los individuos comienzan a controlar de manera conciente su fecundidad, lo que marca el comienzo de la etapa III de la transición demográfica en la cual la tasa de nacimientos disminuye de manera gradual hacia el equilibrio con una reducida tasa de muertes. La reducción de la fecundidad, de manera típica, queda rezagada de la disminución de la mortalidad ya que aquella no puede darse hasta que se debilitan las instituciones económicas y sociales que apoyan la fecundidad y en tanto no emerjan nuevas instituciones que favorezcan una reducción de la fecundidad a niveles más congruentes con los bajos niveles de mortalidad. Los teóricos de la transición demográfica explican la adopción de esta nueva familia pequeña, "ideal", en relación con las transformaciones industriales y urbanas del siglo xIx. Las actividades industriales y urbanas parece que modifican de manera sustancial el papel de la familia en la producción, consumo, educación y recreación. La reducida importancia de la familia debilita las presiones sociales que favorecen una fecundidad elevada, ya que es a través de la extendida familia agraria como se canalizan muchas de estas presiones en la sociedad. El valor económico de los niños disminuye por el crecimiento de la educación masiva o compulsiva, que rescata a los niños de la fuerza de trabajo potencial. La gente llega a percibir que la mortalidad (en especial la infantil) ha disminuido de manera sustancial, y que se requieren menos nacimientos para lograr un cierto tamaño de familia con hijos vivos.

A través de esta multitud de grandes transformaciones, las presiones por una elevada fecundidad se debilitan y la idea del control conciente de la fecundidad cobra fuerza(4) En las primeras etapas no se requiere

- La adopción de esta idea no es uniforme a través de todos los segmentos de la sociedad. De manera usual es adoptada inicialmente por la élite, cuya situación social hace que esté mejor enterada del cambiante medio social y le permite también tener mayor acceso al conocimiento de los métodos requeridos para el control de la fecundidad eficientes de manera razonable. 
tecnología muy elaborada para el control de la fecundidad, aunque existe un mayor conocimiento y uso efectivo de métodos populares tales como el coitus interruptus, el aborto y varios dispositivos burdos. Después, surgen demandas por medios más efectivos y menos objetables de control de la fecundidad, lo que lleva al desarrollo de métodos más modernos.

\section{Estado actual de la teoría}

Como se observó antes, la teoría de la transición demográfica constituye en esencia una descripción plausible del complejo fenómeno social y eco- $\rightarrow$ nómico que tiene lugar en el siglo xIX en Europa. Es notable en tales componentes la ausencia de teorías, de mecanismos de "causación""suscepti-" bles de especificarse y de medirse una escala de tiempo definible. Sin embargo, se han generado algunas hipótesis muy generales las cuales han sido - confirmadas por sucesos posteriores, por ejemplo, la proposición de que la mortalidad por lo general responde de manera más rápida que la fecundidad a las fuerzas de la medicina y del desarrollo y la predicción hecha en los años cincuenta de que en regiones tales como Asia podían esperarse por lo tanto grandes incrementos de población en los decenios siguientes:

Sin embargo, es notable que sólo se han hecho esfuerzos limitados para examinar las proposiciones más específicas y "explicativas" de la teoría de la transición, en especial aquellas que se refieren a los factores asociados con la disminución de la fecundidad europea. Los estudios más intensivos de este tipo han sido realizados en años recieńtes en la Oficina de Investigación de la Población en Princeton, bajo la dirección del profesor Ansley S. Coale. Este "Estudio sobre la Fecundidad Europea" constituye un examen sobre bases comunes del proceso de declinación de la fecundidad en más de 700 provincias de Europa, lo cual permite comparaciones internacionales y generalizaciones para el continente en conjunto. La mayoría de los estudios nacionales de que se compone aún no han sido terminados, pero la información disponible proporciona abundantes oportunidades para un examen empírico preliminar de la teoría de la transición. Sólo puede proporcionarse aquí un estracto de los resúmenes. Una crítica más extensiva e intensiva se presenta en otro trabajo de Coale. ${ }^{6}$

Un hallazgo importante de los estudios de Princeton, es que los niveles globales de fecundidad en la Europa de pretransición estaban lejos de ser - 1 uniformes y en cambio variaban de manera considerable de provincia a provincia y de país a país. Esto se debió en parte a las diferencias en las - pautas de la nupcialidad, pero cuando este factor es controlado persisten grandes variaciones en la fecundidad - los niveles más bajos de fecundi-

3 Véase por ejemplo la discusión en Notestein mencionada en la nota ${ }^{8}$, pp. 22-23.

- A. J. Coale en Proceedings of the IUSSP. International Population Conference, Lieja, Bélgica, Unión Internacional para el Estudio Científico de la Población, 1973, pp. 53.72. 
dad son sólo dos tercios que los más elevados. A esto se puede ofrecer una gran variedad de explicaciones. Dos posibilidades son que la fertilidad (definida en demografía como la capacidad biológica para reproducirse), se vio afectada por normas nutricionales ${ }^{2}$ o por la costumbre, duración e intensidad de la lactancia, 8 o por ambas. Otro competidor importante es que algunas subpoblaciones pretransicionales estaban de hecho practicando el control de la fecundidad de manera conciente 9

Los estudios de Princeton fundamentan con claridad la importancia que tiene para el nivel global de fecundidad, en Europa, las proporciones de

* casadas. Las normas europeas occidentales del matrimonio, ${ }^{10}$ del matrimonio diferido y del celibato extensivo, presentan agudo contraste con los de Europa Oriental, y de manera aún más marcada cuando se comparan con las pautas universales más antigüas y más próximas de matrimonio en muchos países actuales de menor desarrollo relativo.

Cuando se cuantifica y examina el racimo de variables socioeconómicas a las cuales la teoría de la transición atribuye la disminución de la fecun- didad en Europa (industrialización, urbanización, educación, declinación de la mortalidad y otros factores), resulta bastante claro que cierto número de proposiciones confiables en algunas versiones de la teoría de la transición son demasiado dóciles. En algunas regiones, como en partes

- de Francia, la fecundidad empezó a declinar antes de que se empezara a extender la industrialización y urbanización y previa o simultaneamente con la reducción de la mortalidad. Ciertos factores socioeconómicos estuvieron asociados con (pero no necesariamente como causa de) cambios demográficos en algunos países, pero en otros estas relaciones no fueron del todo claras. En realidad, en la mayoría de los países los datos mues- tran agrupaciones regionales subnacionales que tienden a corresponder más a grupos lingüísticos y culturales que a las variables socioeconómicas que son importantes para la teoría de la transición. De aquí que el modelo causal propuesto por esta teoría ha probado ser más evasivo con los datos empíricos que con las proposiciones teóricas.

Coale ${ }^{6}$ resume la generalización de la teoría de la transición que subsiste en términos de tres amplias precondiciones para una declinación sustancial de la fecundidad dentro del matrimonio:

${ }^{7}$ R. E. Frisch y J. W. McArthur, Science, 1974, Núm. 185, p. 949.

${ }^{8} \mathrm{La}$ evidencia de este aspecto ha sido reseñada por J. Knodel y E. van de Walle, Population Study, 1967, Núm. 21, p. 109. Informa que en sus datos para Alemania la correlación elevada entre lactancia y fecundidad casi desaparece cuando se introduce el ajuste estadístico por mortalidad infantil, con lo cual se acentúa la duda en cuanto al impacto demográfico de las prácticas de lactancia.

${ }^{3}$ Véase por ejemplo: N. E. Himes, Medical History of Contraception, Baltimore, Williams \& Wilkins, 1936; P. Demeny, en Population and Social Change; D. V. Glass y R. Revelle (Comps.), Russak, Nueva York, Arnold, London and Crane, 1972, pp. 153-172; D. E. Dumond, Science, 1975, Núm. 187, p. 713; y. D. E. C. Everseley en Population in History, D. V. Glass y D. E. C. Everseley (Comps.), Chicago, Aldine, 1965 , pp. 46-52.

${ }_{10}$ Véase J. Hajnal en Population in History, D. V. Glass y D. E. C. Everseley (Comps.), Chicago, Aldine, 1965, pp. 101-143. 
1) La fecundidad debe estar entre los cálculos de la elección conciente. Los padres potenciales deben considerar esto como un modo de pensamiento aceptable y de forma de conducta para sopesar las ventajas y desventajas antes de decidir tener otro hijo - de otro modo, por ejemplo, la mayoría de los menonitas del presente podrían considerar inmorales tales cálculos y, en consecuencia, no controlar la fecundidad marital;

2) Una fecundidad reducida debe ser (vista como) ventajosa. Las circunstancias sociales y económicas percibidas deben hacer que la fecundidad reducida parezca ventajosa para las parejas individuales;

3) Deben estar disponibles técnicas efectivas de control de la fecundidad. Deben ser conocidos los procedimientos que en realidad prevengan los nacimientos, y debe haber suficiente comunicación entre los esposos así como el apoyo para que ambos puedan emplearse productivamente.

Los datos para Europa muestran que un elevado desarrollo en esencia era suficiente para establecer estas precondiciones y lograr que declinara la fecundidad en Europa. Sin embargo, no existe evidencia sobre los niveles "umbral" de desarrollo que se requieren para que esto suceda y es evidente que las precondiciones para que decline la fecundidad existen en situaciones de un reducido desarrollo social y económico, como en algunas partes rurales de Francia y de Hungría. ${ }^{11}$

\section{IMPORTANCIA DE LA TEORÍA DE LA TRANSICIÓN DEMOGRÁFICA PARA PAÍSES EN DESARROLI.O}

En las actuales condiciones de los países en desarrollo, la importancia de la teoría de la transición es un importante tema científico y político. Desde un punto de vista científico, la debilidad y ambigüedades de la teoría, para explicar las experiencias europeas, puede estudiarse con nuevos detalles si se observa el proceso demográfico actual y futuro de los países en desarrollo. Por ejemplo, es posible que las fallas en la explicación de la teoría para Europa se puedan deber en parte a la falta de datos adecuados sobre factores potencialmente importantes, dado que la explicación de la tendencia a disminuir de la fecundidad, no se contempló en los tiempos en que estos datos se recolectaron. La aplicación de las técnicas modernas de medición demográfica y socioeconómica, en sentido

"La llamada "hipótesis del umbral" (sustentada en Population Bolletin, 1963, Vol. 7, Núm. 134), se encontró que era también deficiente cuando se sometía a la r prueba empírica con datos de países europeos (véase E. van de Walle y J. Knodel, en Contributed Papers of the IUSSP Sydney conference, Lieja, Bélgica, Unión Internacional para el Estudio Científico de la Población, 1967, pp. 47-51. Debe enfatizarse que, aún cuando niveles muy elevados de desariollo parecen ser suficientes para establecer las precondiciones para que decline la fecundidad marital, no necesariamente sobreviene tal disminución. Por ejemplo, en los países de la URSs en Asia Central tales como Tadjikistán. Turkmenistán y Uzbekistán, han persistido elevados niveles de fecundidad marital hasta 1970, y en verdad los niveles de 1970 pueden ser más altos que los de 1926 (A. J. Coale, comentario personal). 
retrospectivo, en los países en desarrollo, puede proporcionar mejores oportunidades para validar o refutar (por lo menos para estos países) hipótesis que se han establecido para las relaciones entre mortalidad, fecundidad, y diversos cambios sociales y económicos. ${ }^{21}$

La importancia de la teoría de la transición en la época moderna puede ser aún de gran interés para la formulación de políticas. Si se puede probar que dentro de un período razonable de tiempo el desarrollo social y económico origina una declinación "natural" de la fecundidad, como se predice por algunos con base en la teoría de la transición, los supuestos subyacentes sobre los principios de población, apoyados por diversos líderes nacionales permitirá que sean verificadas. Por otra parte, si resulta que la teoría de la transición (cualquiera que sea su validez para Europa) no es apropiada en las condiciones de los países modernos de escaso desarrollo, serán muchos los supuestos revisados para que los dirigentes as. piren a plantear la política correcta sobre los principios de población.

Cuando se considera la importancia de la teoría de la transición en los países actualmente en desarrollo, las dificultades explicativas de la teoría general están compuestas por diferencias bastante sustanciales entre los países en desarrollo y la Europa del siglo XIX, en algunas de las variables socioeconómicas y demográficas importantes para la teoría. La situación es de carácter mixto: en algunos aspectos las circunstancias diferentes de los países en desarrollo, sugieren serios obstáculos para que se complete la transición de manera oportuna a través de la declinación "natural" de la fecundidad como en las condiciones europeas. En otros aspectos, estas diferencias constituyen razones para anticipar la configuración de las etapas que señala la teoría de la transición en estos países con una rapidez desusada.

Entre las diferencias que actúan de manera acumulativa en contra de \& la disminución "natural" y oportuna de la fecundidad en los países en desarrollo, se encuentran por lo menos los siguientes:

- al) El ritmo y la fuente del descenso de la mortalidad. Las disminuciones en la mortalidad de los países europeos fueron graduales y, por lo general, estuvieron relacionadas con las fuerzas económicas y sociales del desarrollo y la industrialización. Esto mismo no es válido para muchos países en desarrollo, donde las reducciones de mortalidad han sido más dramáticas y, a menudo, han sido el resultado principalmente de tecnologías importadas, que pueden ser transferidas con relativa facilidad y están relacionadas sólo en forma marginal al ritmo y nivel general de desarrollo. El resultado es que los niveles de mortalidad en los países en desarrollo son mucho más bajos que los de la temprana Europa industrial, aún cuando los factores que los indujeron pudieran ser más o menos naturales y por lo tanto tener un menor impacto sobre la fecundidad;

T2 Véase la explicación de estas posibilidades en R. Freedman, Population Index, 1965 , Núm. 31, p. 425 . En una publicación reciente se presenta un intento interesante por aplicar la teoría de la transición demográfica sobre bases retrospectivas con datos de América Latina (véase S. E. Beaver, Demographic Transition Theory Reinterpreted, Lexington, Mass., Lexington Books, 1975). 
a2) Los niveles de fecundidad antes de su disminución. En la mayoría de los países en desarrollo de la actualidad, la fecundidad es mucho mayor que en la Europa de pretransición. Por ejemplo, a principios del siglo XIX la tasa de nacimientos en Inglaterra se ha estimado que había sido por lo general menor a 35 por mil, mientras que en muchos países en la actualidad (como Tanzania e Irán) es superior a 45 por mil, y en algunos (como Afganistán) posiblemente de más de 50. Estas elevadas tasas de nacimientos se deben de manera principal a la práctica casi generalizada, en la mayoría de los países en desarrollo, del matrimonio a edades jóvenes, en contraste con las prácticas extensivas en la Europa del siglo XIX en que se posponía el matrimonio a edades avanzadas o no se realizaba;

a3) Migración internacional. Los efectos adversos de un crecimiento muy rápido de la población, en un país dado, pueden en teoría ser mitigados por la migración internacional. En el siglo xIX, los países de Europa "exportaron" decenas de millones de ciudadanos a los "nuevos" continentes de América y Oceanía, o a otras partes de sus colonias en el exterior. Con todo, las realidades políticas y económicas actuales indican que la migración internacional no constituye ya una salida para el crecimiento excesivo de la población;

a4) Tasa de crecimiento de la población. Como resultado de las diferencias señaladas, todas las cuales operan en la misma dirección, el crecimiento de la población en la mayoría de los países en desarrollo es literalmente extraordinario - sin precedentes en la experiencia humana, incluidas las de Europa en la época de transición. Las tasas de crecimiento de tales países son de un orden tan elevado como $3.4 \%$ (por ejemplo en Argel, Colombia y República Dominicana, entre otros), con una media de $2.5 \%$. Una población dada duplicaría su tamaño, a la primera tasa, en sólo 20 años (y podría incrementarse 32 veces en un siglo) y con un ritmo de crecimiento como el de la última tasa, la duplicación tardaría menos de 30 años. En contraste, los países europeos que estaban en transición demográfica, rara vez duplicaron su población en menos de 50 años y la media fue de alrededor de 90 años. Por lo tanto, el no puntua. lizar sus transiciones hizo que los países europeos mantuvieran las tasas de población afines al desarrollo en demanda de necesidades básicas y de inversión total, que son ahora enfrentadas de manera cotidiana por los dirigentes de muchos países de menor desarrollo relativo. Tales demandas a menudo compiten por recursos naturales claves, como la de inversión en industria y agricultura y pueden así contribuir a retrasar el desarrollo social y económico. El molesto efecto circular puede ser lento o aun contravenir cualquier tendencia "natural" subyacente que conduzca a la transición demográfica que resulte de las fuerzas del desarrollo;

a5) Impulso para el crecimiento futuro. El rápido crecimiento de los países en desarrollo será más difícil de frenar que el reducido crecimiento de la transición en Europa. Como resultado de su fecundidad, mucho más elevada, los países en desarrollo modernos han tenido una estructura de edades mucho más "jóven" que la que tenía Europa y por lo tanto un 
impulso mucho mayor para el crecimiento futuro. Por ejemplo, aún en el caso improbable de que la fecundidad declinara en los países en desarrollo dentro del siguiente decenio, para la "restitución" del nivel ahora característico de los países desarrollados, la población del mundo con menor desarrollo relativo continuará creciente durante 60 a 70 años, y para el año 2050 habrá alcanzado un tamaño cercano a $90 \%$ mayor que su nivel de 1970. Si la fecundidad de reposición es aplazada hasta 20 años después, el incremento sería superior a $150 \%$. Para países específicos de menor desarrollo relativo, con estructuras de edades excepcionalmente jóvenes, la fuerza del impulso del crecimiento bajo estos dos supuestos sería todavía grande; $;^{13}$

a6) Oportunidades de movilidad ocupacional y del campo a la ciudad. La movilidad ocupacional fuera de la agricultura y la migración espacial de las áreas rurales a las urbanas, proporciona oportunidades alternativas de vida para la creciente población rural durante la transición europea. ${ }^{1+} \mathrm{El}$ incremento natural, mucho más rápido, de los modernos países de menor desarrollo, presenta graves dificultades para proveer oportunidades de ocupación y movilidad espacial comparables. El incremento en la demanda de empleos no agrícolas a menudo excede el incremento de la oferta y, las extraordinarias tasas de rápido crecimiento urbano, en muchos de los países en desarrollo, amenazan arrollar sus capacidades de acomodo. Es cierto que en algunos casos las políticas se esfuerzan por reducir los flujos de migración rural-urbana, aún cuando se mantiene el predominio de la población rural, con lo cual se limitan los efectos de la vida urbana sobre la familia y los valores ante la reproducción establecidos como hipótesis; ${ }^{15}$

a7) Menores oportunidades de participación de la mujer en la fuerza de trabajo. Existe alguna evidencia de que en Europa, la entrada creciente de la mujer en la fuerza de trabajo no agrícola fue un factor en la disminución de la fecundidad. Si esto es cierto, la tasa más rápida de crecimiento natural de los países en proceso de desarrollo de la actualidad, implica que la entrada de la fuerza de trabajo está creciendo más rápido también. En condiciones de ceteris paribus, esto tendería a limitar el crecimiento de la demanda de trabajo femenino;

i: Por ejemplo, bajo los dos supuestos alternativos descritos, la población de Nigeria en el año 2050 pudiera incrementarse entre 108 y 205\%; la de Bangladesh en 125 a $248 \%$; y la de México entre 118 y $229 \%$, respectivamente (véase Teitelbaum. Foreign Affairs, 1974, pp. 747-749).

"Véase por ejemplo K. Davis, Population Index, 1963, Núm. 29, p. 345.

$\therefore$ Además, las pautas de urbanización observadas en algunos países de menor desarrollo relativo, difieren en varios aspectos importantes respecto de otros países del mismo nivel de desarrollo y de los de Europa del Siglo xIx; de ahí que la urba. nización no puede representar la misma fuerza para medir el cambio en todos los casos. Véase, por ejemplo, la explicación en The Determinants and Consequences of Population Trends, Nueva York, Naciones Unidas, 1973, Vol. I, pp. 97-98. Véase también S. Kuznets, Proceedings of American Philosoplyy Society, 1974, Vol. 118, Núm. 1, p. 1 . 
a8) Dificultades para proporcionar educación generalizada. Con cohortes de edad escolar que se duplican sólo cada dos o tres decenios, muchos países de menor desarrollo relativo están experimentando claras dificultades para expandir las facilidades educativas a una tasa lo suficiente rápida para proporcionar oportunidades de educación a la creciente proporción de población elegible. Esto significa el aplazamiento de la meta de educación universal, junto con los efectos hipotéticos sobre la conducta de la fecundidad en la transición.

También existen diferencias entre la situación de la transición europea y la de los modernos países en desarrollo, los cuales actúan en favor de una declinación más rápida y expedita de la fecundidad en los países en desarrollo.

b1) Ritmo de desarrollo social y económico. Para muchos países de menor desarrollo relativo, el desarrollo social y económico ha sido más rápido que en la Europa del siglo xIx. En la medida en que tal desarrollo "ocasione" o acelere la disminución de la fecundidad marital, implica también que se complete de manera más rápida la transición;

b2) Métodos de control de la fecundidad. La disminución de la fecundidad en Europa parece haberse debido a la utilización creciente y más efectiva de medidas de control de la fecundidad tales como el coitus interruptus y el aborto, aunque para los estándares modernos la efectividad del primero no fue muy alta y el peligro de la última fue grande. En la actualidad se ha desarrollado tecnología contraceptiva y técnicas seguras de aborto y posibilidades adicionales tales como la esterilización volun. taria segura y aceptable, disponibles por primera vez en la historia. A cualquier nivel dado de motivación hacia el control de la fecundidad, es probable que el accesa a tales métodos perfeccionados conduzca a una práctica más frecuente y efectiva y en consecuencia con mayores efectos demográficos;

b3) Mayor libertad para la diferición del matrimonio y el incremento del celibato. En Europa la transición demográfica empezó en el contexto del matrimonio tardío y del celibato extensivo. En los países en desarrollo, en la actualidad, por lo general y casi de manera universal el matrimonio se efectúa a edad temprana. Por lo tanto, aún cuando la transición en Europa durante el siglo XIX contó de manera fundamental con una reducción de la fecundidad marital (en verdad los matrimonios ocurrían a edad temprana y resultaban más frecuentes, lo cual tendió a incrementar la fertilidad total), en los países en desarrollo la fecundidad puede reducirse mediante cambios en las prácticas y de la fecundidad dentro del matrimonio, ${ }^{16}$ y al menos no tendería a incrementarse como resultado de matrimonios tempranos y elevadas frecuencias maritales;

1" La política de población de la República Popular de China, en apariencia concede gran importancia al aumento de la edad al matrimonio (véase A. Faundes y T. Luukkainen, Study Family Planning, 1972, Núm. 3, p. 172; J.S. Aind, en People's Republic of China: An Economic Assessment, Washington, D.C., Joint Economic Committee, Congreso de los Estados Unidos de Norteamérica, 18 de mayo de 1972 , 920. congreso, segunda sesión, pp. 301-316. 
b4) Creciente legitimidad de la norma de familia pequeña. En el siglo XIX, Ia familia grande fue considerada como "normal" en la mayor parte de Europa; existían poco ejemplos obvios de alternativas, y en cualquier caso la difusión cultural fue relativamente limitada. En contraste, el "efecto demostración" de la transición europea dio legitimidad actual a la norma de la familia pequeña y evidencia de la factibilidad de realizarla y diversos factores (tales como la comunicación internacional perfeccionada y la expansión del comercio) han acelerado el proceso de difusión cultural e intelectual;

b5) Interés creciente y capacidad de planificación del gobierno. Las tasas de crecimiento de la población prevalecientes en Europa durante el siglo XIX, elevadas para los estándares históricos (aunque bajas para los estándares del siglo $\mathrm{xx}$ ), no se consideraban excesivas. Los dirigentes modernos están mejor enterados que sus predecesores del siglo XIX, de la importancia fundamental del crecimiento rápido de la población como variable del desarrollo; y los gobiernos de países que representan $81 \%$ de la población del mundo de menor desarrollo relativo (aunque constituyen una minoría de tales países) han declarado que las tasas de crecimiento de su población es excesivạ. ${ }^{17}$ En adición, en la actualidad muchos países en desarrollo cuentan con economistas y planificadores capaces, en principio, de prever problemas futuros, mientras que tal planificación, a nivel nacional era poco conocida en Europa durante el siglo XIX;

b6) Capacidad gubernamental para eliminar barreras lingüístico/culturales regionales. La evidencia del Estudio de Fecundidad Europea de Princeton, demuestra la importancia de las barreras subnacionales linguiístico/culturales en la limitación o en el retraso de la tendencia de la transición demográfica europea. Muy diferentes de Europa del siglo XIX, la mayoría de los actuales países en desarrollo cuentan con la infraestructura administrativa y tecnológica (por ejemplo, planificación y administración central, publicación difundida en diversos lenguajes, transporte perfeccionado, sistemas de telecomunicación, radio, televisión y correos) que les permiten traspasar mejor tales barreras;

b7) Asistencia internacional. La disponibilidad actual de asistencia internacional (a través de Naciones Unidas y otros organismos) significa que los gobiernos de los países en desarrollo pueden invocar la cooperación sustancial de recursos y expertos del exterior. Tal asistencia, si se emplea de manera apropiada, puede reducir las demoras en el proceso de desarrollo que de lo contrario resultarían de la escasez nativa de capital, materias primas, tecnología y "Know-how";

b8) Ritmo más rápido de descenso de la fecundidad. Kirk ${ }^{18}$ y Beaver,"1! entre otros, han observado que los países que han experimentado reducciones de fecundidad en los últimos decenios, muestran un ritmo más rá-

1; Véase Mauldin et. al., loc. cit., p. 392.

${ }^{18}$ D. Kirk, en Rapid Population Grow'h, Washington, D.C., Academia Nacional de Ciencias, 19.71, pp. 123-147.

19 Véase Beaver, op. cit., p. 89. 
pido de disminución que los de Europa en el siglo xix. Este fenómeno puede atribuirse a la combinación cambiante de los factores descritos antes, en especial al desarrollo más rápido, la declinación severa de la mortalidad, un mayor interés y capacidad del gobierno y a la disponibilidad de contracepción efectiva, aborto con seguridad y esterilización.

\section{RESUMEN Y CONCLUSIONES}

Cuando se analiza en forma sistemática la fecundidad europea con los datos disponibles al finalizar el siglo XIX, se verifican empíricamente algunas de las principales proposiciones de la teoría de la transición, pero sólo a un elevado nivel de generalidad. .

Desde un amplio punto de vista macrohistórico, es correcto decir que durante dos siglos, desde mediados de 1700 en adelante, se registraron disminuciones importantes y difundidas en la fecundidad marital, que estuvieron asociadas a importantes transformaciones sociales y económicas en las sociedades europeas. Sin embargo, las proposiciones más específicas y explicativas derivadas de la teoría de la transición no son suficientes para explicar todos los rasgos notorios de la experiencia europea. En particular, la teoría falla en la explicación de fenómenos fundamentales tales como las variaciones en los niveles de fecundidad en la etapa de pretransición, en la determinación del tiempo en que principió y el ritmo de la disminución de la fecundidad, la cual eventualmente se vuelve universal.

Un estudio reciente sugiere que la teoría de la transición, puede alcanzar de manera similar un nivel moderado de éxito en América Latina, en especial cuando la teoría tradicional se modifica sustancialmente y se introducen en adición variables causales, incluidos factores culturales. De nuevo, la teoría (modificada) logra un poder predictivo considerable a un alto nivel de generalidad - aquellas países de América Latina con ni-. veles de desarrollo relativamente altos, tienen más probabilidad de experimentar el inicio de una disminución de fecundidad que otros. Sin embargo, de nueva cuenta, como en el caso europeo, se tiene poco éxito en la predicción del tiempo en que se inicia y la tasa de disminución de la fecundidad con base en la teoría de la transición."2n

Por lo tanto, a niveles altos de generalidad, la estructura causal básica de la teoría de transición parece cierta. Cuando el proceso de desarrollo se alcanza a los altos niveles logrados en Europa y en algunas partes de América Latina, esto parece ser suficiente para establecer las precondiciones para la disminución de la fecundidad. Sin embargo, no puede decir-

\footnotetext{
$\because$ Beaver, ibid, en especial, pp. 145-152. Por desgracia, en este estudio sobre las pautas de fecundidad en América Latina no se separan los efectos de la nupcialidad de los de la fecundidad marital, lo cual puede crear problemas al trabajar con algunos datos de América Latina. El nivel de análisis también es muy agregado, o séa el de estado-nación en oposición al nivel de provincia como el utilizado en los estudios de Princeton sobre la declinación de la fecundidad en Europa.
} 
- se que esto es necesario para tal fin, dado que estas precondiciones también aparecen en situaciones de un exigüo desarrollo económico y social.

Las características socioeconómicas y demográficas diferentes de manera sustancial de los actuales países de escaso desarrollo relativo, deja poca duda de que la disminución de la fecundidad seguirá pautas que difieren de manera marcada de las europeas. No obstante, no hay razón para pensar que un nivel elevado de desarrollo prueba ser menos suficiente o más necesario para el establecimiento de las precondiciones como para que decline la fecundidad; en realidad existe alguna evidencia de que han ocurrido disminuciones de la fecundidad en áreas tanto de alto nivel de desarrollo como en las de bajo nivel.

Sin embargo, debe reconocerse que los elevados niveles de desarrollo de Europa y de partes de América Latina, no constituyen una perspectiva realista para muchos países en desarrollo, al menos en los cercanos años o decenios siguientes; cuando la teoría de la transición demográfica se aplica a tales circunstancias falla en la solución de respuestas adecuadas a dos preguntas de significado científico y político fundamentales:

I) Suficiencia: ¿son suficientes los moderados niveles de desarrollo a los cuales pueden aspirar de manera realista los países de escaso desarrollo relativo, en el mediano plazo, para establecer las precondiciones para que de manera "natural" decline la fecundidad?

2) Oportunidad: si se verifican tales disminuciones, ¿ocurrirán lo suficiente rápido y a un ritmo tal para compensar la disminución aguda de la mortalidad y los elevados niveles iniciales de fecundidad de estos países, en comparación con los de transición en Europa?

La discusión internacional actual gira sobre estas cuestiones de suficiencia y oportunidad. Aquellos que argumentan que el desarrollo "cuidará" de la población piensan que el desarrollo es suficiente ( $\mathrm{y}$ también necesario) para impulsar más o menos de manera adecuada la reducción de la fecundidad en un tiempo aceptable. Los que pugnan por las políticas y programas de población voluntarios (en adición a, no sustitutos de esfuerzos máximos en la esfera del desarrollo), enfatizan la importancia del desarrollo, pero desconfían de su suficiencia y oportunidad para muchos países y por lo tanto invocan esfuerzos directos para acrecentar y acelerar su impacto demográfico. Los que apremian políticas obligatorias de población, aceptan la importancia del desarrollo, apoyan las políticas que alientan las limitaciones voluntarias, pero sostienen que ninguna de las dos es suficiente ni adecuadamente rápida para satisfacer la apremiante necesidad. Todos están de acuerdo con la proposición general de la teoría de la transición en el sentido de que niveles elevados de desarrollo social y económico de manera eventual tendrán efectos reductores sobre la fecundidad. Sin embargo, la estructura de estas posturas teóricas es piramidal: de manera ortodoxa cada hallazgo científico se apoya en diferentes aspectos de la misma base.

Si esto es así, bien podría preguntarse por qué el debate acerca de la importancia actual de la teoría de la transición es tan estridente, tan ca- 
rente de objetividad científica. La respuesta es una conocida de política, desde el último decenio: quiérase o no, la población se ha convertido en un asunto importante de interés internacional. En esta arena, las perspectivas que se derivan de consideraciones políticas, más que doctorales, han sido ocultadas con el manto científico de la teoría de la transición.

En principio, la politización de la población no es mala. En verdad, si las políticas deben ser tomadas en cuenta, tendrían que serlo vía el proceso político. Pero la politización no es amiga de la ciencia, en particular cuando la evidencia empírica es ambigua y puede interpretarse como respaldo de diversas perspectivas políticas, ${ }^{2 !}$ como sucede con la teoría de la transición. En la actualidad, en el campo de la política de población, las decisiones deben responder de manera principal a las prioridades y restricciones políticas, más que a las demandas de validez científica. En particular, en una interpretación simplista, es clara la gran virtud política de la teoría de la transición al sostener que en todas las circunstancias el desarrollo se "hará cargo" de la cuestión de la población. No obstante, los científicos están obligados a informar, con base en el examen detenido de la teoría de la transición en su perspectiva histórica y actual, a los encargados de establecer las políticas, que cometerían un gran desacierto al adoptar tal punto de vista simplista y determinístico. ${ }^{2-2} \mathrm{Al}$ proceder con el problema entrelazado de la población y el desarrollo, el desafío que enfrentan científicos y políticos es conseguir una buena comprensión de los procesos de la transición demográfica en toda su complejidad y sutileza, con lo cual se busca una base más informada para la política.

21 Los científicos han tenido bastante experiencia con la aplicación "fácil" de evidencia inadecuada a la política social para cuestiones de política. En correspondencia, basta mencionar los casos del darwinismo social, de la eugenesia y el Lysenkoinísmo y los más recientes excesos en las diferencias de subpoblación.

* En verdad puede argumentarse que no es común que la teoría de la declinación de la fecundidad proporciona líneas definitivas de política. Para una exposición sobre esto véase T. K. Burch, Sociology, 1975, Vol. 54, Núm. 1. 